\title{
Le symbolisme dans la gastronomie française et indonésienne : Revue interculturelle
}

\author{
${ }^{1}$ Tania INTAN, ${ }^{2}$ Nurul Hikmayaty SAEFULLAH, ${ }^{3}$ Ferli HASANAH, \\ ${ }^{4}$ Vincentia Tri HANDAYANI \\ 1,2,3,4 Département de français, Universitas Padjadjaran - Indonésie
}

Reçu le 5 avril 2019 | Accepté le 4 décembre 2019

\begin{abstract}
RÉSUMÉ. La gastronomie est un élément inséparable de la vie humaine. Elle possède sa propre cosmologie qui s'attache à la culture d'une nation. Comparer deux cultures différentes vers la gastronomie ouvre un nouveau paradigme de réfléchir pour recueillir un point de vue sur le monde puisqu'il s'agit de la communication interculturelle qui amène aux étudiants au savoir-vivre côte à côte. Cette étude tente donc d'analyser l'interculturalité pour résoudre les problèmes de la compréhension culturelle à partir des termes culinaires en français et en indonésien ainsi que les valeurs comprises. La recherche est menée en utilisant la méthode descriptive-qualitative, en observant les données d'une manière linguistique et culturelle. Comme les données viennent de cultures différentes, il est pratiqué donc de mesures comparatives. Les résultats de cette recherche montrent que la gastronomie comprend des symboles et qu'elle prend une position pertinente dans la conception culturelle surtout dans la formation de stéréotypes basée sur la langue. Ce résultat d'analyse n'est qu'un simple exemplaire de l'étude interculturelle qui propose encore de possibilités à développer.
\end{abstract}

Mots-clés : interculturalité, gastronomie, symbolisme.

\begin{abstract}
Gastronomy is an inseparable element of human life. It has its own cosmology that ties into the culture of a nation. Comparing two different cultures towards gastronomy opens up a new paradigm of thinking to gather a point of view on the world since it is about intercultural communication which brings students to life skills side by side. This study therefore attempts to analyze interculturality in order to solve the problems of cultural understanding from culinary terms in French and Indonesian as well as the values understood. The research is conducted using the descriptive-qualitative method, observing the data linguistically and culturally. As the data come from different cultures, there are therefore comparative measures. Research results show that gastronomy includes symbols and that it takes a relevant position in cultural conception, especially in the formation of stereotypes. This finding of the analysis is only a simple copy of the intercultural study which still has possibilities to develop.
\end{abstract}

Keywords: interculturality, gastronomy, symbolism.

面 auteur correspondant : tania.intan@unpad.ac.id

Pour citer cet article (Style APA) : Intan, T., et al. (2019). Le symbolisme dans la gastronomie française et indonésienne : Revue interculturelle. Francisola: Revue Indonésienne de la langue et la littérature françaises, 4(2), 138-149. doi: 10.17509/francisola.v4i2.24205. 


\section{INTRODUCTION}

La langue et la culture sont des choses inhérentes. Dans la situation globale $\mathrm{d}$ 'aujourd'hui, l'apprentissage de langues et de cultures étrangères sont très importantes, surtout quand nous les relions avec le concept de l'interculturalité. Cela veut dire que nous apprenons un élément étranger pour mieux comprendre soi-même. La dimension de culture dans l'étude de linguistique devient une partie fondamentale pour enrichir et élargir la connaissance des apprenants de langues et de cultures étrangères. Le but de l'étude est surtout pour la communication et en plus pour changer le paradigme de penser du point de vue ethnocentrique à l'ethno-relatif.

L'influence de la culture dans notre rapport à la langue est bien plus importante qu'on ne le pense (Chauvot, 2016). La langue est un symbole unique représentant tout ce que l'on considère important. Certains mots peut-être existent dans quelques langues mais dissimulent des autres langues. Cela veut dire que le vocabulaire d'une langue symbolise la nature de sa propre culture. Il est évident, d'après l'hypothèse de SapirWhorf (cité dans Rahardi, 2006 p.60), que la culture domine la structure du processus de penser. Ce postulat est renforcé par la théorie de Koentjaraningrat (2009 p. 186-187) sur la forme de la culture. Il exprime qu'il y a trois formes de la culture: (1) la forme de la culture comme l'idée, le concept, les valeurs ou les normes; (2) la forme de la culture comme l'activité de l'homme dans la société ; et (3) la forme de la culture comme les objets de travail de l'homme. L'activité et l'interaction de l'homme dans la société construisent un système. Elles font une organisation de sorte que le peuple vive dans une bonne circonstance. Parlons maintenant de la coutume ; les coutumes pratiquées dans la société sont, entre outre, comment nous parlons (la langue) et comment nous mangeons (la gastronomie).

De la recherche précédente (Intan, T., Wardiani, S.R., et Handayani, 2014), il est connu que dans la plupart des têtes orientales, des éléments concernant de la gastronomie se positionnent inséparablement des systèmes de la religion et la société. Ainsi que l'opinion occidentale, y compris les Français en général, qui assument la vision que leur culture culinaire devient un indice de l'identité héréditaire qu'on pourrait s'en fier partout. En principe, la gastronomie n'est seulement pas des repas ordinaires, mais des magies qui possèdent d'une grande valeur, un symbole, des règles, et une représentation de mode de consommation et de production des gens impliqués. C'est pourquoi, actuellement il y aurait toujours des traditions qu' on ne considère pas usées ou inutilisables, malgré le grand changement de la valeur sociale, elles sont même notées comme exotiques, et donc économiques.

Nous avons discuté la relation inévitable entre la culture et la langue, ainsi que la culture et la cuisine, dans une même culture. Cependant, nous pourrions aussi parler de la coutume des cultures différentes, en comparant des langues ou bien ces cuisines. Cela nous montre que nous pouvons faire la recherche sur les deux matériaux en seule problématique du point de vue de l'interculturalité. Quand on dit l'interculturalité, c'est de voir « la réciprocité dans les échanges et la complexité dans les relations entre cultures " (Clanet, 1993 p. 21). Basé sur cette idée, nous allons discuter dans cet article la problématique suivante : «comment les valeurs culturelles sont-elles comprises dans les termes gastronomiques françaises et indonésiennes?». De cette problématique, nous avons l'objectif de de faire une revue sur l'interculturalité dans les termes gastronomiques français et indonésiens en voyant les valeurs culturelles depuis ces termes.

La recherche sur l'interculturalité nous emmène à un nouveau point de vue sur le monde. Il s'agit de nous montrer un autre aspect de la culture, de l'autre culture. Dans ce regard, nous sommes obligés de comprendre ce que l'on appelle "le génie locale ». Ce terme au début est présenté par Quaritch Wales dont la définition est «la capacité de la culture locale face à des influences culturelles étrangères au moment de la culture liée » (cité dans Rosidi, 2011 p. 29). Sungsri (2010) décrit le génie local en 
autre mot comme «connaissances et expérience reliées à la vie de tous les jours, les occupations, et la culture ont été transmises de génération en génération ».

Parler de la culture c'est parler de ses codes. Les codes culturels se manifestent dans tous les aspects de vie qu'on étudie dans la sémiologie. Littlejohn (2009, p. 53) explique que la sémiologie comprend des théories des codes, des signes et des symboles représentant l'objet, l'idée, la condition, la situation et le sentiment hors de ces signes. Il s'agit de connaître des sens des signes et de les interpréter puisque l'on sache la façon du destinateur construit le message. Le concept de sens s'enchaîne aux valeurs idéologiques et le concept de culture où sont les symboles créés. Barthes (cité dans Kriyantono, 2007, p.268) met en relief «l'interaction entre le texte et l'expérience personnelle et culturelle de son usager et l'interaction entre la condition dans le texte et la condition souhaitée par l'usager ». Ailleurs, l'étude sémiologique de Barthes expose: (1) la dénotation qui parle du vrai sens ou le phénomène vu par les cinq sens; et (2) la connotation qui est les sens culturels apparus suit la construction culturelle.

La recherche des symboles culturels présents dans la gastronomie française et indonésienne découvre aussi l'aspect sémiologique en dehors de l'interculturalité. Selon la culture orientale, les questions relatives à l'alimentation et à la médecine ne peuvent être séparées du système de croyances et du système socioculturel. De même, les cultures occidentales, y compris chez les Français en général, considèrent toujours leurs traditions culturelles culinaires comme les identités héréditaires de l'identité et en sont fières. Ce n'est pas seulement une cuisine ordinaire, mais aussi quelque chose qui contient des valeurs, des symboles, des règles, et des modèles de consommation et de production communautaire. Cette recherche est donc faite à partir de la recherche précédente par Intan, Wardiani, et Handayani (2014) intitulé « Le Folklore dans la tradition culinaire française » et la première partie de cette recherche discute les expressions utilisant les termes culinaires.

\section{MÉTHODE}

La recherche se déroule du mois de juillet jusqu'au octobre 2016. Elle procède par une décomposition du sujet, dans ce cas, les échantillons sont des expressions du domaine culinaire en français et en indonésien. Cette méthode considère les choses par rapport à l'ensemble de ses éléments constructifs. L'analyse se fait d'une manière qualitative (identifier, classifier, interpréter, commenter) où l'on s'accorde les données descriptives avec les résultats que nous distribuons en phrases descriptives.

Le concept d'analyse qualitative selon Bogdan et Biklen (1982) est le procès d'organiser les données et de les classifier, de trouver une formulation et de la présenter au public. Drury cité par Moleong (2013) explique les étapes de l'analyse qualitative, telles que : étudier les données en trouvant les mots clés et les idées essentielles, analyser les mots clés et essayer de trouver les thèmes, composer un modèle, et donner le code, par exemple avec l'énumération et la classification.

Le travail est concentré sur les symboles dans les termes gastronomiques français et indonésiens. Comme cela va impliquer deux cultures différentes, la recherche entre donc dans le domaine comparatiste. Les données sont recueillies par l'observation des expressions françaises et indonésiennes dans des dictionnaires et Internet. Pour les valider, nous faisons l'interview aux informateurs, donc les natifs français et indonésiens (professeur de français qui est aussi anthropologue).

Les données sur l'expression culinaire française et indonésienne sont recueillies et groupées par les légumes, les fruits, les épices, et les graines. Les données sont ensuite analysées en plusieurs étapes. Tout d'abord, une analyse de la signification des expressions, qui se concentre sur le lexème culinaire contenu dans la phrase. L'analyse de cette signification examine la référence lexique dans l'expression. Ensuite, l'analyse sémiotique est faite pour obtenir les valeurs mythiques qui y sont contenues. À la suite d'une analyse du niveau linguistique, l'évaluation se poursuit sur l'analyse 
interculturelle par la confrontation du symbolisme culturel français et indonésien soutenu par la connaissance des aspects anthropologiques, historiques, géographiques, et botaniques.

Le déroulement de recherche commence en observant les expressions françaises et indonésiennes dont les mots clés sont la gastronomie. Après, les données sont triées et nous avons les termes gastronomiques, qui sont classifiés selon les matières, l'avantage, et la valeur. La recherche suit également la méthode analytique puisque l'on touche les résultats attendus. Les analyses de données sont faites en considérant le point de vue anthropologique, historique, géographique et botanique. L'intérêt des anthropologues pour étudier la nourriture est causé de la réalité que les problèmes alimentaires se produisent non seulement des raisons physiologies, mais aussi pour celles de culturelles. Quelque chose d'acceptable ou rejeté comme nourriture est sous contrôle culturel. Selon Levi-Strauss (cité par Maryetti, 2007), la nourriture occupe une place essentielle dans la pensée humaine pour deux raisons. L'existence des êtres humains et tous ses attributs peuvent être définis au moyen de la cuisson. En outre, cuisiner et manger de la nourriture marque la transition de la nature à la culture.

Afin d'avoir une vision plus claire, nous présenterons dans cet article quelques exemples de données et d'analyses avec l'étude du champ de sens du terme langue culinaire en français. En décrivant la signification de chaque donnée, le Grand dictionnaire indonésien (KBBI) est utilisé pour les données d'expression indonésiennes et le dictionnaire œcuménique dans le réseau en ligne (Ortolang, 2005) pour les données d'expression françaises.

\section{RÉSULTATS ET DISCUSSION}

A propos de la culture orientale, les questions relatives à l'alimentation et la médecine ne peuvent pas être séparées du système de croyances et du système de socioculturel. En outre, dans ce cas, le point de vue de la culture occidentale n'est pas différent, y compris les Français en général. On suppose toujours que leur culture culinaire est une identité qu'ils peuvent montrer partout dans le monde et dont ils sont fiers. La gastronomie n'est pas seulement une cuisine ordinaire, mais aussi quelque chose qui contient des valeurs, des symboles, des règles et des modes de consommation y compris la production.

Dans la vie moderne, il y a des choses traditionnelles qui ne sont pas nécessairement considérées comme obsolètes ou vétustes. En raison d'un changement, le sens comporte une valeur exotique et même économique. À l'échelle mondiale, il y a également un changement de valeurs pour revenir à la nature, comme les efforts pour populariser l'eau minérale comme le retour de boissons, l'utilisation des plantes médicinales pour guérir les maladies naturellement, et les cosmétiques. Cette condition a finalement conduit au renforcement de la notion d'identité culturelle dans le cadre du système de sécurité sociale dans la culture de l'application donne une signification positive à l'économie.

Dans la perspective de la diversité culturelle, la gastronomie est une identité, la représentation et la production de la culture qui se sont développées dans la communauté. La nourriture et ses types peuvent décrire le comportement de la vie telle que la santé, le style de vie, l'environnement, et les systèmes sociaux. De cette façon, c'est clairement illustré que la gastronomie est une représentation de la résistance de la communauté avec un large éventail de signification. En outre, elle montre aussi le contexte social, l'économie, et le type de groupe de consommateur. Par conséquent, la société gastronomique parfois gérée par les règlements coutumiers qui contiennent des conseils, l'abstinence, et les procédures éthiques d'utilisation (MelionoBudianto, 2004, p. 65).

Concernant la compréhension de la culture en général, d'après Duhaime (2016), les produits alimentaires souvent identifiés par le symbolisme de la vie humaine. En général, par exemple, le fruit est connu 
comme un symbole de vie d'amour et de l'humeur, au contraire, les légumes sont considérés comme ayant une relation avec les conditions matérielles de l'homme, le travail et la vie sociale. Cette formule montre essentiellement des relations interculturelles qui montrent des similitudes à la fois la différence entre les Français et le mode de vie indonésien.

Selon la vision anthropologique, la nourriture de l'homme se compose d'animaux et de plantes qui sont obtenues des alentours. Autrefois, en tant que chasseurs et cueilleurs dans les temps anciens, les gens se déplacent d'un endroit à l'autre, suivent la direction de la chasse, et aussi pour tenter de se sauver d'autres chasseur humain. Pendant ce temps, on a tenté de gouter les jeunes feuilles, les fruits, les graines et les tubercules qui poussent le long du chemin. De l'expérience éprouvante, on a élu les types de matériaux qui peuvent être utilisés. Contrairement aux animaux qui peuvent se sauver lors de la chasse, les plantes ne peuvent se déplacer, de sorte que le moyen pour se défendre est une variété de composés qui sont formés dans son corps. On a trouvé donc, il y avait un goût amer, acide, abrasif, ou sucré et salé dans le corps de la plante. De ces saveurs que les hommes choisissent ceux qui conviennent à son goût.

La disponibilité de ces plantes est certainement différente d'un endroit à l'autre. Ce qui est clair, si un pays est situé près de l'équateur où le soleil brille toute l'année, les plantes peuvent vivre toute l'année, donc la garantie de la disponibilité alimentaire. Mais ce n'est pas le cas des pays dans des zones qui ont l'hiver comme la France. Nombreuses espèces animales et végétales ne vivent pas en cette saison. C'est pourquoi les gens qui vivent dans ces régions consomment des espèces végétales et animales qui sont différents du pays tropique.

Quand les humains ont commencé à se stabiliser, leurs besoins de base ont augmenté et ne se limite plus à la nourriture. Conformément au développement, les gens commencent à avoir besoin de médicaments, des herbes pour la cuisine, des matériaux pour construire une maison, des fibres pour les vêtements, même des fleurs pour embellir l'environnement. Tous ces besoins peuvent être obtenus à partir d'une variété d'espèces végétales. Ainsi, un certain nombre de plantes peuvent être cultivées en fonction des besoins humains. La cultivation a été pratiquée différemment, selon le climat, la fertilité des sols, disponibilité de l'eau, la richesse de la flore et la faune locales. Pour cette raison, la production agricole varie géographiquement.

Sastrapradja (2012, p. 19) argumente que le développement agricole mondial ne peut pas être séparé des parcours des nations européennes. Par exemple : les Portugais, les Espagnols et les Néerlandais qui parcouraient des différentes régions de la terre qui donc permet la propagation des espèces végétales d'une région à l'autre. Par conséquent, il y a beaucoup d'aliments principaux de l'Amérique latine repartent dans diverses parties du monde, ainsi que des plantes de la Chine continentale et l'Inde se propagent aux pays d'Amérique centrale, et l'Amérique du nord. Pendant ce temps, les pays européens utilisent les pays d'Asie et de l'Afrique en tant que colonie, et ils transportent des produits de grande valeur économique en Europe comme le café, le thé, la cannelle aux colonies.

Dans le cadre du folklore non-verbale, il existe des liens entre des ressources alimentaires et ses ingrédients, les cultures, les traditions, et les coutumes. Pour déterminer l'identification et la classification d'expression culinaire qui a été faites sur l'analyse précédente, on peut voir que la gastronomie, en particulier sous la forme de produits alimentaires. La nourriture est divisée en légumes, fruits, herbes, et les grains, dont l'identification vient de la matière, les avantages, et la valeur. Dans les sections ci-dessous, il y aura une discussion sur certains produits alimentaires dans diverses revues comme un soutien d'étude interculturelle.

\subsection{Les légumes}

Dans la vie quotidienne, beaucoup de types de légumes sont consommés selon le goût. A part de son utilisation pour les 
besoins alimentaires de l'homme, Crystallia (2015) argumente que des légumes ont une valeur symbolique qui varie en fonction du type (légumes verts, légumes secs, feuilles de légumes ou tubercules). Dans la culture européenne, les pois, les pois chiches, les haricots et le brocoli expriment comme un signe que la vie humaine se déroule selon le plan d'ego. C'est pourquoi il y a souvent un sentiment de frustration et d'insatisfaction. Le poireau a aussi sa propre conception. Il est un symbole de puissance désirée. Alors que les pommes de terre symbolisent le courage dans le travail. Cela est compréhensible, parce que la pomme de terre est un producteur de glucides. Dans les pays tempérés froids comme la France, ses habitants consomment beaucoup de pommes de terre, qui sont généralement consommés avec de la viande, du poisson ou du poulet et des légumes. Les pommes de terre ont d'abord été cultivées en Amérique du Sud, puis a été introduit par le portugais et l'espagnol en Europe. Ensuite, Les Hollandais l'ont emmené en Indonésie, mais dans ce pays, les pommes de terre sont considérées comme un légume plutôt que comme un substitut pour le riz.

La carotte est un signe d'un manque d'énergie de la vie, alors que le navet symbolise la déception inévitable au niveau de l'ego. Le terme «carotte» provient de néerlandais qui signifie la racine. Ayant grandi avec les hommes depuis plus de mille ans, les racines des plantes de carotte évoluent également suite à l'appétit de l'homme. Non seulement la taille d'une racine qui s'élargie, mais la racine aussi dure que le bois a lentement gonflé et contenu de l'eau. Les légumes ont d'abord été cultivés en Afghanistan et en Asie centrale il y a environ 5000 ans.

\section{Donnée 1}

Les carottes sont cuites.

D n.f / pf v.pas.

« Tout est parti ; pas d'espoir»
L'expression 'les carottes sont cuites' décrit la situation quand il n'y a plus d'espoir. Cette expression française est apparue au début du XXe siècle et résultait d'un autre terme plus long (vers le XIXe siècle) avoir ses carottes cuites «mourantes ». Le mot « carotte » dans la phrase ne fait pas référence à la signification ultime (la vie), mais à la cuisson qui donne un sens, c'est-àdire à la cuisson. Selon une autre interprétation, la comparaison avec les carottes cuites provient d'autres régions qui traitent les carottes en bouillie. Jusqu'à ce que le sens ultime soit une ruse pour tirer un avantage d'une personne. En revanche, à partir du vocabulaire de la carotte, on ne peut expliquer le sens de la perte ou de la perte et sa relation avec le verbe cuire " cuisinier ». En effet, en français, le mot cuire (v) «cuisinier » est conjugué dans le passé et a été fait.

Au XVIIe siècle, les carottes étaient des légumes bon marché, souvent utilisés comme nourriture pour les pauvres. Idéalement, les carottes sont cuites avec de la viande, mais à ce moment-là, les carottes ne sont consommées qu'en période difficile. En raison de sa forme et de sa paronymisation presque identiques, carotte est associée au mot crotte (n.f) « saleté », jusqu'à ce que le phiait des carottes semble décrire une personne souffrant de constipation. Carotte (n.f) « carotte » a donc la valeur de significations péjoratives, pour décrire quelque chose qui est endommagé, pourri, impie ou moins bon. La signification péjorative pour le mot carotte continue de croître, qui est alors associée à la mort. Quand une famille pauvre ne vit que de la nourriture sous forme de carottes ou ne peut manger que des carottes, ce qui signifie vraiment économiser de l'argent, cela signifie que la famille est en train de mourir (Technologies, s.d).

\begin{tabular}{lcc}
\hline & Les carottes sont cuites & Il n'y a plus aucun espoir \\
\hline Cuisine à la carotte (ingrédient principal) & + & - \\
Rien d'autre prévu & - & + \\
Conditions remplies & + & + \\
\hline
\end{tabular}


Les carottes sont le deuxième légume le plus consommé en France après les pommes de terre (Interfel, s.d.). Comme il est facile à obtenir, les carottes sont largement consommées. Cette commodité est ensuite associée à la nourriture de bon marché, de sorte que les personnes qui ne peuvent pas se permettre de faire des carottes comme ingrédient alimentaire principal. La situation qui a donné naissance aux carottes n'est plus un espoir.

\subsection{Les Fruits}

Dans toutes les cultures du monde, ainsi que des fleurs, des fruits signifient le bénéfice. Il implique l'excitation, la joie et un sentiment d'amour. Le fruit est associé à la saison. Son état pourrait symboliser un caractère : cru, mûre, doux, amer, arôme particulier, la couleur, etc. Chaque fruit a sa propre caractéristique.

Dans presque toutes les cultures, les fruits peuvent également contenir des connotations sexuelles. Par exemple, dans la culture française, pomme rouge implique la passion et les tentations du monde. La pomme est un défi mémorable, doux et parfumé, ce qui illustre les défis pour les hommes. Autre couleur de la pomme, par exemple, le vert, signifie le sens de l'amour innocent (Beccia, 2014, p.13).

Le citron, jaune ou vert, montre la situation complexe (acide) dans la zone affective. Son goût profond et ne termine que par des événements extrêmes. Dans la mythologie grecque, associée à Vénus et Adonis, quand Adonis est mort, Vénus pousse un arbre, pour se souvenir des dieux, qui est rempli de fleurs blanches et de citron comme un symbole de fidélité. En outre, les oranges sont devenus le symbole de la réussite de l'amour qui ne connaît pas la fin, qui apporte toujours le bonheur et la chaleur dans le cœur. Alors que dans la culture indonésienne, les connotations sexuelles sont plus attachées aux légumes avec certaines formes telles que l'aubergine, le concombre, etc.
Certains fruits, dans la culture française, est également associée aux aspects de la religiosité comme mentionné dans la Bible. Par exemple, la poire est exprimée un symbole de bonnes réalisations d'un effort spirituel. Elle peut également signifier la grâce. Le raisin sec est le synonyme de bonheur et de joie, les deux peuvent se produire simultanément. Alors, la saveur causée par l'ivresse du vin est la preuve. Symboliquement, le vin présente une brève période de satisfaction humaine sur le plan affectif. La couleur rouge de cerise fait référence au culte du Christ, en particulier dans son sang. De plus, la couleur de ce fruit dans les emblèmes de céleste rappelle à la vie éternelle qui fait référence à la Vierge Marie. Cependant, à part de la signification spirituelle, les cerises peuvent aussi être un symbole de la sensualité et de passion.

\section{Donnée 2}

Travailler pour des prunes

$\mathrm{vp} p+\mathrm{d}$.f. $/ \mathrm{pl}$.

«Travailler pour gagner un peu d'argent»

Étant donné que le «prune » (n.m) ne se réfère pas toujours au fruit. En France centrale, à partir du XIIIe siècle, les prunes pourraient également signifier : frapper dans l'expression "il s'est pris une prune», un pruneau dans «il commence à flancher »; et chanceux (en termes de négociation) ou de malchance selon le contexte; " ne pas valoir prune » signifie non faisable, et « ne preisier /prisier une prune» veut dire ne respecte personne.

Ce dernier sens qui devient alors la référence changeante de "prune» pour rien 'pas de résultat, pas d'usage'. Cette expression vient du siège de Damas par les Croisés au XIIe siècle. Le siège a échoué. Quand ils sont retournés en Europe, ils n'ont apporté le butin sauf la prune pourpre de la ville de Damas. Les prunes sont amenées en France avec l'intention d'être dégustées ensemble. Les rois qui sont déçus de leur défaite sont certainement fâchés de voir ses soldats « juste " porter des prunes. Dans la ville, les prunes poussent partout, abondamment. Donc, la prune est considérée comme quelque chose qui n'est pas spéciale. 


Pas de résultat, pas d'utilisation
L'effort pour obtenir la prune
Cela n'a pas d'importance
La tentative échouée (le résultat n'est pas comparable)

\begin{tabular}{ll}
+ & - \\
- & + \\
+ & + \\
+ & + \\
\hline
\end{tabular}

Prune (n. f) est le nom du fruit qui fait partie du genre Prunus, le sous-un de Prunus. Le sous-genre se distingue des autres sous-genres (y compris les pêches, les cerises, et autres) parce qu'il a une pièce terminale et que ses côtés sont isolés, non regroupées en une section, les fleurs sont groupées 1-5 dans une branche courte. Le fruit est violet rougeâtre, contient beaucoup d'eau et a un goût sucré. Ce fruit est connu des Européens depuis 200 ans. Dans l'histoire, au moment de la deuxième croisade de 1150, les soldats sont rentrés chez eux après un échec. Alors qu'ils assiégeaient la ville de Damas, la ville productrice de prunes, ils faisaient face à une population défensive, essayant de les expulser de la ville. Apporter des prunes est une forme de consolation, car la guerre se termine par un résultat inattendu (Camille, 2010). Ainsi, l'expression 'travailler pour des pruneaux' fait référence à la signification pour des résultats minimes ou inexistants.

\subsection{Les épices}

D'après Demichel (s.d.), des épices principalement sont utilisées en raison de ses trois aspects : en raison de son goût (utilisé dans la cuisine), ses avantages thérapeutique (utilisés en médecine), et en raison de son arôme (utilisé pour conserver des cadavres comme dans momification en Égypte). Les Grecs utilisent principalement des épices sous la forme de feuilles qui sont produits localement.

Dans la croyance occidentale, l'ail a la capacité de purifier, nettoyer le sang. De plus, l'ail peut aussi expulser les insectes. L'assaisonnement est très utile pour renforcer la confiance, et peut aussi bannir un fantôme. L'oignon rouge a également fonction de la purification et le nettoyage, son énergie est dérivée du monde surnaturel, et est facilement contrôlé. Ce matériau est souvent utilisé pour la magie. La région est considérée comme le lieu d'origine de l'oignon est l'Asie centrale, tandis que l'ail de Chine (Ensminger, 1994). L'ail et les oignons sont obligatoires dans la cuisine indonésienne.

Le piment n'est pas favorisé par les Français parce qu'il est trop épicé. Les Français ont obtenu un goût épicé de poivre dans la nourriture. Alors qu'en Indonésie, le sambal comme un dérivé de la production de piment est toujours servi à la table du dîner (Sastrapradja, 2012, p. 72). En raison d'un penchant pour ce goût poivré, les indonésiens cultivent aussi tant de variétés. Ses résultats sont le piment rouge, piment vert, piment frisé, le piment gendot, etc.

La coriandre symbolise le côté tendre et généreux du feu, utilisé pour la fertilité, l'amour, et le mariage. En plus de ce matériel est également utilisé pour tirer la douceur et les avantages des choses différentes. Alors que les feuilles laurier impliquent l'élément feu, et a un côté magique qui apporte l'atmosphère de clair, pur, et la guérison. Les feuilles de laurier sont également considérées comme un symbole de la victoire.

Le gingembre est la racine ou le rhizome qui contient l'énergie d'un élément fort de feu, augmenter la passion d'une manière durable. Très utile pour doubler la force et la vitesse de l'activité humaine. D'autres épices, c'est la vanille qui apporte douceur chaude de l'élément de feu, elle est une source d'énergie positive. La vanille est largement utilisée pour augmenter la passion de l'amour et de bonté, si crédible comme une offrande à la déesse.

Le principal ingrédient de l'assaisonnement est le sel, dont l'existence est devenue obligatoire dans la vie humaine. D'autres avantages du sel sont pour conserver les aliments (Tradysel, s.d.). Le sel est censé préserver le corps humain, alors il est considéré comme purifier le corps, comme mentionné dans la Bible, que les villes de Sodome et Gomorrhe bombardés 
avec du sel. Puis, une légende révélée que le marin qui viole les lois de la nature peuvent transformer en une statue de sel. En outre, $c^{\prime}$ est un mythe qu'on sème le sel dans la cuisine pour échapper à la malchance et malédictions.

\section{Donnée 3}

Mettre son grain de sel

v. d. n.m. p n.m.

«Interférer avec non inviter»

La phrase dérivée du latin cum grano salis, est apparue au XXème siècle. Le sens dénotatif de cette expression est de donner un grain de sel, tandis que le sens figuratif « un grain de sel " fait référence à la signification d'aider, de donner son opinion. $\mathrm{Au}$ XIIIe siècle jusqu'au XVIIIle siècle, le sel était inestimable. Le prix élevé du sel fait que les Français conservent l'usage du sel pour la cuisine. Le sel devient une épice de cuisine très chic. C'est pourquoi, dans l'expression utilisée, le seul déterminant de la personnalité du grain des grains est "un grain de sel ", ce qui signifie que le sel est calculé par grain. En fait, les cellules «salées " sont incluses dans les noms non comptabilisés. Cependant, à ce moment, l'expression signifie connotatif négatif. Mettre son grain de sel signifie répondre à la conversation sans y être invité où intervenir dans une affaire non sollicitée (Bob, s.d.). Cette expression utilise un style métaphorique in absentia, un élément qui n'est pas présent. Le terme « sel » de la cellule culinaire (n) produit son propre goût afin qu'il ait une signification positive, tandis que l'on compare l'implication d'une personne dans une conversation qui change l'atmosphère inconfortable qui a une signification négative.

\begin{tabular}{lll}
\hline & Intervenir dans une conversation & Mettre son grain de sel \\
\hline soudainement interférer dans & + & - \\
une conversation & & + \\
améliorer le goût en cuisine & - & + \\
donner du changement & + & + \\
\hline
\end{tabular}

Le sel est un composant majeur dans la cuisine transformée, car le sel donne un goût salé aux aliments. Le mélange du sel dans la cuisine donne certainement son propre goût. Cette situation est décrite dans l'expression mettre son grain de cellule « interférant dans une conversation non invitée ». La présence salée du sel peut changer le goût de la cuisine. Quand quelqu'un s'engage soudainement dans une conversation non sollicitée, alors la conversation sera plus colorée, peut avoir un certain effet.

\subsection{Les céréales}

Le pain qui est devenu l'aliment de base des Européens est un symbole de l'attitude de partage et aussi le symbole de la vie elle-même. Le pain est la nourriture la plus souvent utilisée comme cadeau aux dieux. Il symbolise la métamorphose et représente le cycle des semences dans le monde végétal, de céréales qui sont transformées en farine et de farine en pain. En autre côté, le pain est également une collaboration entre trois professions importantes dans le processus de production : les agriculteurs, le moulin à farine et du pain (l'agriculteur, le meunier et le boulanger). Le pain est le plus fondamental de l'alimentation humaine, qui devrait être obtenu facilement. Cependant, en réalité, les hommes doivent travailler assez dur pour l'obtenir. Par conséquent, le pain peut être un symbole de l'esprit de travaille, les espoirs et les plaisirs de la vie. Le blé lui-même est un symbole de la fécondité, la vie et la résurrection.

En Indonésie, le riz est la nourriture principale, notamment le riz blanc, bien qu'il y ait aussi le rouge foncé et même le noir. La disparition des variétés locales en raison du système d'importation et la promotion de la technologie élimine également la manipulation de la récolte de la culture et de la transformation du riz traditionnel (Sastrapradja, 2012, p. 36). Alors qu'autrefois, le riz est très vénéré et respecté jusqu'à ce qu'il existe les diverses cérémonies, et la 
figure déesse Sri comme la protectrice des agriculteurs. Le riz dans les rizières qui sont près de la récolte sera vu courbé (pas droit) en raison de la charge des grains de riz. En d'autres termes, le riz est de plus en plus comme un arc. Dans la vie sociale, lorsqu'une personne a une connaissance qualifiée, la richesse, le rang et la position, elle doit imiter le riz qui est modeste.

Le maïs est une culture très importante qui est connu pour sa vitesse de croissance, dont le nombre peut être produit, ainsi que sa capacité à réfracter la lumière. Le maïs n'est pas une plante sauvage, il a besoin $\mathrm{d}$ 'intervention humaine dans la plantation et l'entretien. Cependant, le maïs est une plante généreuse, assez pour se nourrir, les hommes vont récolter les résultats rapidement. D'après (Sastrapradja, 2012, p. 43), les experts estiment que le maïs de l'Amérique centrale, et seulement autour du 16ème siècle, a été présenté à la population de l'Asie du Sud-Est, y compris l'Indonésie, par les Portugais. Pour les gens qui vivent dans les zones pluviométrie faible, comme Madura et Nusa Tenggara, le maïs est largement cultivé et consommé comme aliment de base. Les jeunes feuilles de maïs sont également utilisées pour l'emballage des cigarettes.

A propos du pain, étant l'aliment de base de nombreuses cultures surtout de l'Occident, cela symbolise non seulement de la vie, mais aussi le travail, le sacré, et le partage (Tekfak, 2016). C'est un aliment très simple mais fondamental, qui allie les quatre éléments fondamentaux que sont la terre (le blé), l'eau, l'air (les bulles issues de la fermentation), et le feu (la cuisson). Ainsi, l'homme a donné d'une multitude d'expressions.

\section{Donnée 4}

Avoir du pain sur la planche

$v p+d$ n.m $p d n . f$

«Beaucoup de travail»

Cette phrase a plusieurs significations du XIXe siècle : " peut vivre sans travailler, pénalités », et finalement déterminé la signification réelle en 1914 « un beaucoup de travail à faire ». Signification de cette expression est différente à la fin du XIXe siècle. Sa signification est que nous avons suffisamment de provisions pour l'avenir. Les agriculteurs préparent le pain en grande quantité, ils économisent sur une planche de bois fixé au plafond. Le pain, peut en effet être stocké pendant une longue période. Maintenant, l'expression implique beaucoup de sens d'origine de travail est apparue au début du XXe siècle. Situé au XIXe siècle, lorsque les rations judiciaires de pain pour les personnes reconnues coupables doivent subir le travail forcé avec un temps assez long. Cette phrase a une longue et difficile référence de sens. Une autre référence indique que la position de pain à bord décrit le processus de fabrication du pain qui doit être stocké sur une plaque de cuisson («Les expressions françaises décortiquées: Avoir du pain sur la planche,» s.d.)

\begin{tabular}{lll}
\hline & Beaucoup de chose à faire & Le pain sur la planche \\
\hline beaucoup de chose à faire & + & - \\
mettre en place pour la coupe & - & + \\
$\begin{array}{l}\text { Prend beaucoup de temps } \\
\text { pour travailler sur }\end{array}$ & + & + \\
\hline
\end{tabular}

Chaque jour, les Français consomment 120 grammes de pain par personne (Chesnais, 2016). Les spécialités françaises souvent utilisées comme menu du petitdéjeuner sont un pain typiquement français, le pain baguette comme illustré dans cette phrase. La baguette est un pain français traditionnel qui est long et a une peau dure, une couleur brune et beaucoup de trous à l'intérieur. Ce pain cuit au four est alors mangé avec un mélange de confiture ou de miel, peut également avec du beurre, du beurre de cacahuète ou du Nutella. Ce pain est populaire depuis la révolution française. En 1793, il y avait un décret qui énonce le même pain pour tous, le pain égalitaire, qui signifie que tout le monde, riche ou pauvre, mange le même pain : habituellement 
baguette a une largeur de 3-4 cm, hauteur 5-6 $\mathrm{cm}$, longueur $70-80 \mathrm{~cm}$ et poids 250 grammes (Tekfak, 2016). La référence à la signification de nombreux emplois est attribuée à la longueur du pain baguette qui, une fois coupé, nécessite une longue durée.

\section{CONCLUSION}

En analysant les données, nous pouvons conclure qu'en général, les expressions françaises contenant les termes gastronomiques expriment le caractère $\mathrm{d}^{\prime} u n e$ personne, décrivent la situation ou la condition physique d'une personne. Tandis que les expressions indonésiennes, elles comportent des conseils ou la situation. De plus, nous pouvons faire une formule que l'on fait une analogie des termes gastronomiques dans les expressions avec les caractères de ces termes, les parfums, les conditions, les positions, les couleurs, les saveurs et les formes.

L'étude comparée du symbolisme en français et en indonésien laisse encore un domaine qui peut être approfondi, par exemple la recherche sur le concept de temps, le sens des animaux, les couleurs, etc. Le phénomène du langage est fondamentalement complexe et multidisciplinaire. Par conséquent, dans le cadre de l'interculturalité, une telle étude est encore très à développer.

\section{REMERCIEMENTS}

Nous tenons à remercier tous les organismes et/ou toutes les personnes qui ont apporté une contribution significative à la rédaction et/ou à l'amélioration de l'article.

\section{RÉFÉRENCES}

Beccia, I. (2014). Vanités. Repéré à musée des beaux-arts de Bordeaux website: http://www.musba-

bordeaux.fr/sites/musba-

bordeaux.fr/files/vanites.pdf

Betrand, D. (2016). Le Symbolisme de la Nourriture et des Boissons. Repéré à L'Arche de Gloire website: https://larchedegloire.com/les-aliments/

Bob. (n.d.). Mettre son grain de sel. Repéré à https://www.languefrancaise.net/Bob/1
8835

Bogdan, R., \& Biklen, S. K. (1982). Qualitative research for education: An introduction to theory and methods. Boston: Allyn and Bacon.

Camille. (2010). Pourquoi dit-on "pour des prunes"? Repéré à Axiom Cafe.fr website: https://axiomcafe.fr/pourquoidit-on-pour-des-prunes

Chauvot, P. (2016). L'Influence de la Culture Autant de Manières de Parler l'Anglais que de Pays. Repéré à Communicaid website:

https://www.communicaid.fr/blog/lang ues/linfluence-de-culture-facon-deparler/

Chesnais, E. de la. (2016). On consomme toujours moins de pain en France.

Clanet, C. (1993). Introduction aux approches interculturelles et en sciences humaines. Toulouse: Universitaires du Mirail.

Crystallia. (2015). Symbolique des fruit et légumes. Repéré à http://crystallia.unblog.fr/symboliquedes-fruits-et-legumes/

Demichel, N. (n.d.). Les aliments symboles: les épices. Repéré à plumart.com website: http://www.plumart.com/vf1300/html/b ody_6113epices.html

Ensminger, A. . (1994). Foods and Nutrition Encyclopedia. In Foods and Nutrition Encyclopedia (Vol. 1). CRC Press.

Intan, T., Wardiani, S.R., et Handayani, V. T. (2014). Folklor dalam Tradisi Kuliner Perancis. Penelitian Hibah Pengembangan Kapasitas Riset Dosen Fakultas Ilmu Budaya. Bandung - Indonésie: Universitas Padjadjaran.

Interfel. (n.d.). Les fruits et légumes frais: Carotte. Repéré à https://www.lesfruitsetlegumesfrais.co $\mathrm{m} /$ fruits-legumes/legumes-racinestubercules-et-tiges/carotte/carte-identite

Koentjaraningrat. (2009). Pengantar Ilmu Antropologi. Jakarta, Indonésie: Aksara Biru.

Kriyantono, R. (2007). Teknik Praktis Riset Komunikasi. Jakarta, Indonésie: Kencana.

Les expressions françaises décortiquées: 
Avoir du pain sur la planche. (n.d.). Repéré à Expressio.fr website: https://www.expressio.fr/expressions/a voir-du-pain-sur-la-planche

Littlejohn, S. W. (2009). Theories of Human Communication. Jakarta, Indonésie: Salemba Humanika.

Maryetti. (2007). Makanan dan Struktur Budaya Minangkabau: Studi Kasus di Nagari Kapau Kecamatan Tilatang Kamang Kabupaten Agam, Sumatera Barat. Yogyakarta.

Meliono-Budianto, V. . (2004). Dimensi Etis terhadap Budaya Makan dan Dampaknya pada Masyarakat. Jurnal Makara, Sosial Humaniora, 8(2), 65-70.

Moleong, L. (2013). Metode Penelitian Kualitatif Edisi Revisi. Bandung, Indonésie: Remaja Rosdakarya.

Ortolang. (2005). Portail lexical : un ensemble de ressource et de données.

Rahardi, K. (2006). Dimensi-dimensi Kebahasaan, Aneka Masalah Bahasa
Indonesia Terkini. Jakarta, Indonésie: Erlangga.

Rosidi, A. (2011). Urang Sunda di Lingkungan Indonesia. Jakarta, Indonésie: Kiblat Buku Utama.

Sastrapradja, S. . (2012). Perjalanan Panjang Tanaman Indonesia. Jakarta, Indonésie: Yayasan Pustaka Obor Indonesia.

Sungsri, S. (2010). Lifelong Learning and Wisdom. International Journal of Lifelong Education, 29(6), 647-649. https://doi.org/http://dx.doi.org/10.1080 /02601370.2010.528261

Tekfak, J. (2016). La baguette française. Repéré à Français authentique website: https://www.francaisauthentique.com/1 a-baguette-francaise/

Tradysel. (n.d.). Le symbolisme du sel. Repéré à Tradysel.com website: http://www.tradysel.com/fr/lesymbolisme-du-sel.html 